\title{
Inhibition of varicella-zoster virus replication by an ethanol extract of Lysimachia mauritiana
}

\author{
SOHEE BAE and YOON-JAE SONG \\ Department of Life Science, Gachon University, Seongnam-Si, Kyeonggi-Do 461-701, Republic of Korea
}

Received October 19, 2016; Accepted March 31, 2017

DOI: $10.3892 / \mathrm{mmr} .2017 .6444$

\begin{abstract}
Varicella-zoster virus (VZV), a ubiquitous human $\alpha$-herpesvirus, is the causative agent of chickenpox and shingles. In the present study, we investigated the antiviral activity of a $70 \%$ ethanol extract of Lysimachia mauritiana (LME) against VZV. LME strongly interfered with the replication of both laboratory and clinical strains of VZV without affecting the viability of MRC-5 cells. Moreover, LME treatment suppressed the expression of an essential viral transactivator, immediate-early 62 protein (IE62), in addition to other lytic genes in the later phases of viral replication. The finding that LME exerts potent inhibitory effects on VZV gene expression and replication supports its potential utility as a therapeutic anti-viral agent.
\end{abstract}

\section{Introduction}

Varicella-zoster virus (VZV), belonging to the $\alpha$-subgroup of herpesviruses, contains a linear, double-stranded DNA (dsDNA) genome encoding 71 open reading frames (ORF) (1). The VZV virion particle is composed of an icosahedral nucleocapsid containing the viral genome, a layer of tegument proteins that surrounds the nucleocapsid and a plasma membrane acquired from cellular membranes with the viral glycoproteins $(1,2)$.

Similar to other herpesviruses, VZV is proposed to enter host cells via binding to receptors or fusion of the viral envelope with cellular plasma membrane followed by endocytosis (1). After entry, the nucleocapsid is transported into the nucleus, and viral gene expression is induced by VZV tegument proteins encoded by ORF4, 10 and 62 (1). Among the viral tegument proteins, the immediate-early (IE) protein 62 (IE62) encoded by ORF62, is a major component and transactivator essential

Correspondence to: Dr Yoon-Jae Song, Department of Life Science, Gachon University, 1342 Seongnam-Daero, Seongnam-Si, Kyeonggi-Do 461-701, Republic of Korea

E-mail: songyj@gachon.ac.kr

Abbreviations: VZV, Varicella-Zoster virus; IE, immediate-early; LME, ethanol extract of Lysimachia mauritiana; MOI, multiplicity of infection; PFU, plaque forming unit; ORF, open reading frame

Key words: varicella zoster virus, antiviral, Lysimachia mauritiana for viral lytic gene expression and replication (2). During lytic replication, VZV gene expression occurs in a temporal cascade involving three classes of genes: IE, early (E), and late (L). IE genes regulate the expression of early and late genes. The $\mathrm{E}$ gene subset encodes proteins for viral DNA replication while L genes encode structural constituents of the virion, including glycoproteins and nucleocapsid proteins $(3,4)$. VZV replication utilizes a rolling circle mechanism and is highly cell-associated $(1,5)$.

VZV is widely distributed worldwide and affects almost the entire human population owing to the high morbidity of infection (1). Primary VZV infection causes chickenpox (also known as varicella), a normal childhood illness concurrent with fever and pruritic vesicular rash (5). During primary infection, VZV inoculates respiratory mucosal epithelium, is transported to the skin by cell-associated virus infection, causes dermal lesions and infects sensory ganglia, where it establishes latent infection (6). Herpes zoster (shingles), caused by reactivation of VZV, provokes severe pain in the area of latently infected ganglia (7).

Several antiviral agents, such as acyclovir, famciclovir, valacyclovir and foscarnet, are currently used to treat VZV-associated diseases $(5,7,8)$. These licensed drugs inhibit viral DNA polymerase and are used in combination with corticosteroids for inflammation and narcotics for pain $(1,9)$. However, the efficacy of these agents to treat chronic VZV infections in immunocompromised individuals is limited because of their adverse effects and emergence of resistant VZV strains (7,9). In addition to antiviral drugs, a FDA-approved live-attenuated VZV vaccine is available for infant immunization (10). However, significant concerns still exist with regard to efficacy of vaccination and prevalence of unvaccinated individuals carrying latent VZV (9). Development of novel antiviral drugs to treat VZV-associated diseases with high efficacy and fewer side-effects therefore remains an urgent unmet medical need. In the present study, we showed that the Lysimachia mauritiana extract exerts antiviral activity against VZV lytic gene expression and replication. Our findings support the potential utility of LME as a therapeutic agent for active VZV infection.

\section{Materials and methods}

Cells, viruses and plant materials. MRC-5 cells were obtained from the Korean Cell Line Bank (KCLB no. 10171). The 
recombinant laboratory pOka strain of VZV (VZV-pOka) expressing green fluorescent protein (VZV-pOka-GFP) and clinical YC01 strain of VZV (VZV-YC01) were kindly provided by Dr Chan-Hee Lee (Chungbuk National University, Cheongju, Korea) (11). Plant material (Lysimachia mauritiana Lam.) and the $70 \%$ ethanol extract used in the present study were collected from Jeju Island in Korea and provided by the Jeju Biodiversity Research Institute (Jeju, Korea; specimen no. JBR-324).

Fluorescence microscopy. Fluorescence was examined and images analyzed using an inverted Nikon TS100-F fluorescence microscope (Tokyo, Japan) equipped with a digital camera and Nikon NIS-Elements microscope imaging software.

Quantification of VZV DNA replication. Total DNA was isolated using an Accuprep Genomic DNA Extraction kit (Bioneer, Daejeon, Korea), and VZV DNA was amplified and quantified in a StepOnePlus Real-Time PCR system (Applied Biosystem, Foster City, CA, USA) using HOT FIREPol ${ }^{\mathbb{E}}$ EvaGreen quantitative PCR (qPCR) mix Plus (Solis BioDyne, Tartu, Estonia). The following primers were used for qPCR: VZV ORF62 forward, 5'-TCTTGTCGAGGAGGCTTCTG-3' and reverse, 5'-TGTGTGTCCACCGGATGAT-3'; GAPDH forward, 5'-CATGAGAAGTATGACAACAGCCT-3' and reverse, 5'-AGTCCTTCCACGATACCAAAGT-3'.

Plaque reduction assay. MRC-5 cells were inoculated with serially diluted VZV-YC01-infected MRC-5 cells and treated with either DMSO or LME at concentrations of 5, 10, 25, $50 \mu \mathrm{g} / \mathrm{ml}$. At 6 days after infection, cells were fixed with $10 \%$ formaldehyde at room temperature for $10 \mathrm{~min}$ and stained with $0.3 \%$ crystal violet. After $5 \mathrm{~h}$, the number of plaques was counted, and the virus titer expressed as plaque-forming units (pfu/ml).

Quantification of VZV transcript expression. VZV transcript levels were quantified using quantitative reverse transcription PCR (qRT-PCR). Total RNA was isolated using a HiGene ${ }^{\mathrm{TM}}$ Total RNA Prep kit (BIOFACT, Daejeon, Korea) and reverse-transcribed into complementary DNA (cDNA) with a QuantiTECT ${ }^{\circledR}$ reverse transcription (RT) kit, according to the manufacturer's instructions (Qiagen, Hilden, Germany). cDNAs were amplified and quantified in a StepOnePlus Real-Time PCR system (Applied Biosystems, Foster City, CA, USA) using HOT FIREPol ${ }^{\circledR}$ EvaGreen qPCR mix Plus (Solis BioDyne, Tartu, Estonia). The following primers were used for qRT-PCR: VZV ORF62 (immediate early, IE) forward, 5'-TCT TGTCGAGGAGGCTTCTG-3' and reverse, 5'-TGTG TGTCC ACCGGATGAT-3'; ORF28 (E) forward, 5'-CGAACACGTT CCCCATCAA-3' and reverse, 5'-CCCGGCTTTGTTAGTTT TGG-3'; gB (L) forward, 5'-GATGGTGCATACAGAGAACA TTCC-3' and reverse, 5'-CCGTTAAATGAGGCGTGACTAA-3'; GAPDH forward, 5'-CATGAGAAGTATGACAACAGCCT-3' and reverse, 5'-AGTCCTTCCACGATACCAAAGT-3'.

Western blot analysis. Cells were collected, fractionated and transferred onto nitrocellulose membranes, as described previously (12). Antibodies specific for VZV IE62 and tubulin were purchased from Abcam (Cambridge, UK) and Sigma-Aldrich (St. Louis, MO, USA), respectively. Enhanced chemiluminescence detection reagent (Pierce, Rockford, IL, USA) and secondary peroxidase-labeled anti-mouse immunoglobulin G antibodies (Amersham Biosciences, Piscataway, NJ, USA) were used according to the manufacturer's specifications.

Cell viability assays. CellTiter-Glo luminescent cell viability assay (Promega, Madison, WI, USA) was performed in keeping with the manufacturer's protocol.

\section{Results}

VZV replication is inhibited by LME. To examine the antiviral effects of the 70\% ethanol extract of Lysimachia mauritiana (LME) against VZV, uninfected MRC-5 cells were inoculated with cells infected with VZV-pOka-GFP at an MOI of 0.1, and treated with either $50 \mu \mathrm{g} / \mathrm{ml}$ DMSO or LME. At $0,24,48$ or $72 \mathrm{~h}$ after infection, GFP fluorescence was measured under a fluorescence microscope. At $72 \mathrm{~h}$ after infection, significantly reduced VZV-pOka-GFP fluorescence was observed in the LME-treated cells (Fig. 1A). For quantitative analysis of VZV-pOka-GFP replication, the relative amounts of viral DNA were measured using qPCR with primers specific for ORF62. Compared to $0 \mathrm{~h}$ after infection, VZV DNA was amplified 170,000- and 280,000-fold at 48 and $72 \mathrm{~h}$, respectively (Fig. 1B; compare $0 \mathrm{~h}$ with 48 and $72 \mathrm{~h}$ ). Relative to DMSO-treated cells, VZV replication was downregulated 3.8- and 4.7-fold in LME-treated cells at 48 and $72 \mathrm{~h}$ after infection, respectively (Fig. 1B), clearly supporting an inhibitory effect of LME on VZV replication.

LME-mediated inhibition of replication of the VZV clinical isolate is dose-dependent. The plaque reduction assay was performed to further investigate the inhibitory activity of LME against the clinical isolate of VZV. Uninfected MRC-5 cells were inoculated with serially diluted VZV-YC01-infected MRC- 5 cells and treated with either DMSO or LME at concentrations of $5,10,25$ or $50 \mu \mathrm{g} / \mathrm{ml}$. Cells were re-treated with DMSO or LME at 3 days after infection. At 6 days post-infection, cells were fixed and stained, and the plaque forming units (pfu) per $\mathrm{ml}$ were determined via counting. Interestingly, LME reduced the number of plaques by 2.3- and 17.3-fold at concentrations of 25 and $50 \mu \mathrm{g} / \mathrm{ml}$, respectively, compared to cells treated with DMSO $(0 \mu \mathrm{g} / \mathrm{ml})$ (Fig. 2). The $50 \%$ inhibitory concentration $\left(\mathrm{IC}_{50}\right)$ of $\mathrm{LME}$ for $\mathrm{VZV}$ at which the number of plaques was reduced by $50 \%$ was calculated as $26.09 \mu \mathrm{g} / \mathrm{ml}$.

To ascertain whether the inhibitory activity of LME on VZV replication was due to effects on cell viability, MRC-5 cells were treated with different concentrations of LME, and the levels of cellular ATP representing the presence of metabolically active cells were measured at 24,48 or $72 \mathrm{~h}$ after treatment using the CellTiter-Glo assay. With prolongation of incubation time, cellular ATP levels were increased, possibly owing to cell proliferation. Interestingly, at $72 \mathrm{~h}$ post-treatment, the viability of MRC- 5 cells treated with $25 \mu \mathrm{g} / \mathrm{ml}$ LME was similar to that of DMSO-treated cells (Fig. 3). Treatment with $50 \mu \mathrm{g} / \mathrm{ml}$ LME slightly reduced MRC-5 cell viability by 33 and $39 \%$ at 48 and 72 h, respectively (Fig. 3). Since LME exerted no significant adverse effects on MRC5 cell viability 
A
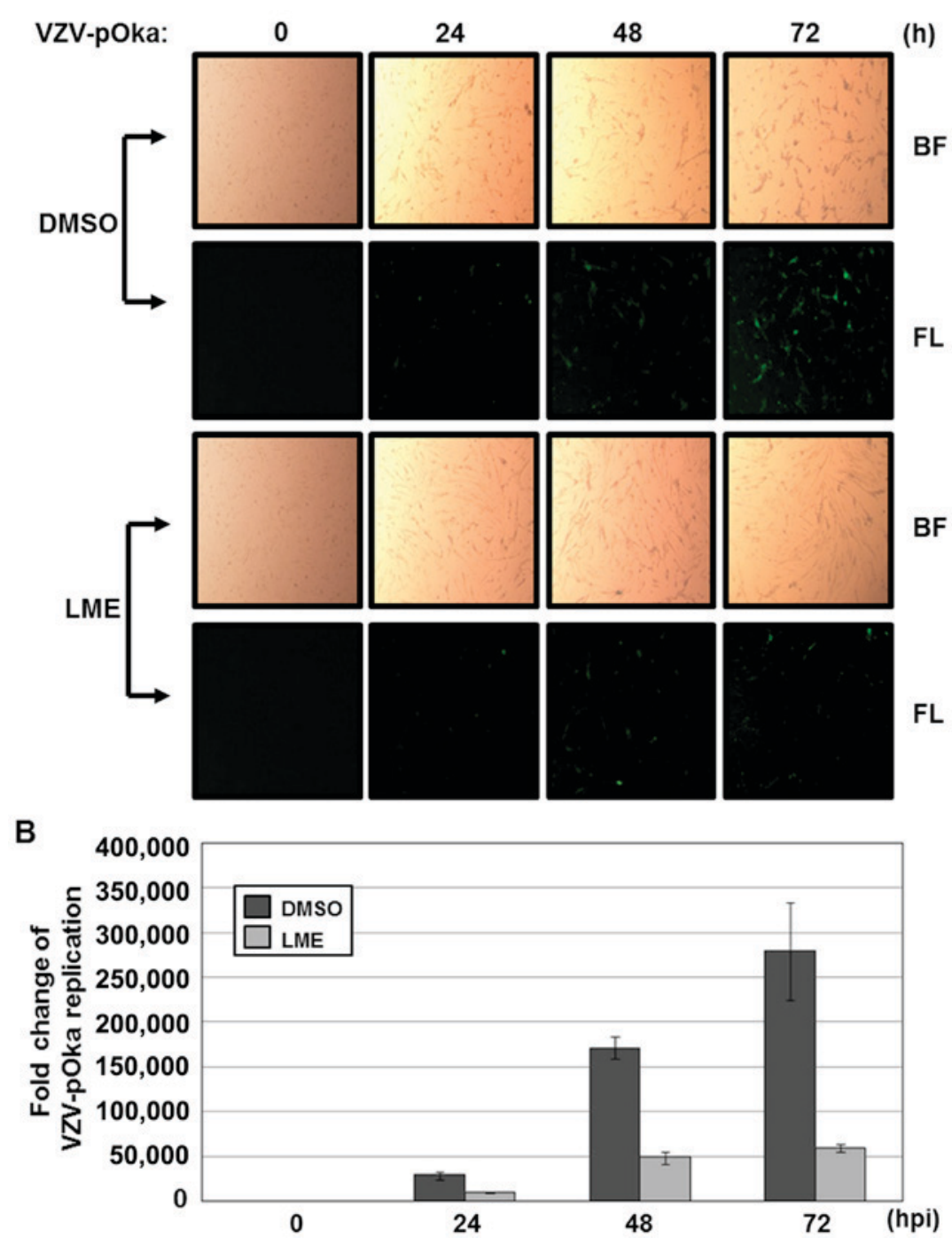

Figure 1. Effects of LME on VZV replication. MRC-5 cells were inoculated with recombinant VZV-pOka-GFP-infected MRC-5 cells at MOI of 0.1 and treated with either DMSO or LME at a concentration of $50 \mu \mathrm{g} / \mathrm{ml}$. (A) GFP fluorescence was monitored under a Nikon TS100-F inverted fluorescence microscope at 0 , 24,48 or $72 \mathrm{~h}$ after infection. (B) Total DNA was harvested and the relative amounts of viral DNA were measured via qPCR using primers specific for ORF62, as described in Materials and Methods. BF, Bright field microscopic image; FL, fluorescence microscopic image; hpi, h post-infection.

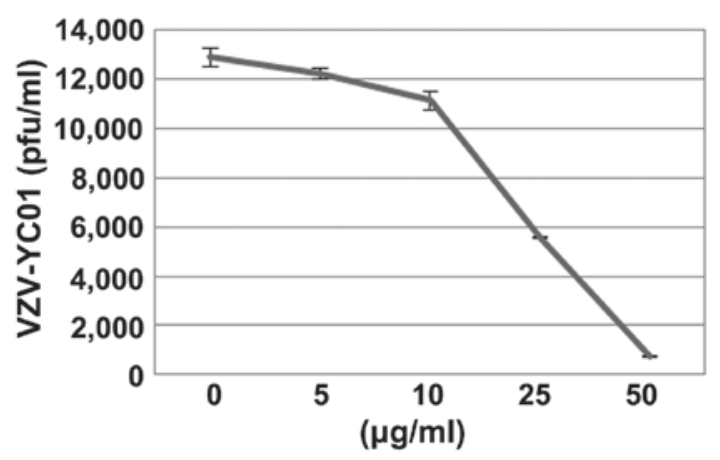

Figure 2. Dose-dependent inhibitory effect of LME on VZV replication. MRC-5 cells were inoculated with serially diluted VZV-YC01-infected MRC-5 cells and treated with DMSO or LME at concentrations of 5, 10, 25 and $50 \mu \mathrm{g} / \mathrm{ml}$. At 6 days after infection, the number of plaques was counted using a plaque assay to determine the titer ( $\mathrm{pfu} / \mathrm{ml})$.

at the $\mathrm{IC}_{50}$ value, it is unlikely that its anti-VZV activity of LME is related to cytotoxicity.
VZV lytic gene expression is downregulated by LME. The effects of LME on VZV lytic gene expression were determined via qRT-PCR analysis of IE, E or L transcript levels. MRC-5 cells were inoculated with VZV-YC01-infected MRC-5 cells at an MOI of 0.1 and treated with either DMSO or LME at a concentration of $25 \mu \mathrm{g} / \mathrm{ml}$. At $0,24,48$ and $72 \mathrm{~h}$ after infection, the relative amounts of transcripts for ORF62 encoding IE62 (IE), ORF28 encoding a subunit of viral DNA polymerase (E) and glycoprotein B (gB) (L) were determined (Fig. 4). In DMSO-treated cells, ORF62, ORF28 and gB genes were induced at $24 \mathrm{~h}$ and higher levels expressed at 48 and $72 \mathrm{~h}$ after infection (Fig. 4). LME had no effect on VZV lytic gene expression at $24 \mathrm{~h}$ after infection (Fig. 4). However, at 48 and $72 \mathrm{~h}$, ORF62, ORF28 and gB transcript levels were significantly reduced in the LME-treated cells (Fig. 4).

The protein level of IE62 encoded by ORF62, an essential transactivator of VZV lytic gene expression, was further determined. MRC-5 cells were inoculated with VZV-pOka-infected MRC- 5 cells at an MOI of 0.1 and treated with either DMSO 


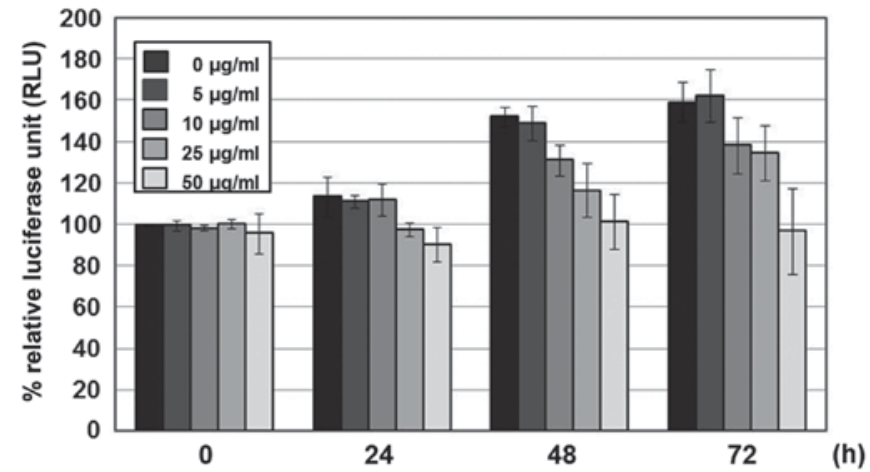

Figure 3. Cytotoxicity of LME against MRC-5 cells. MRC-5 cells were treated with $0,5,10,25$ and $50 \mu \mathrm{g} / \mathrm{ml} \mathrm{LME}$, and cell viability was determined at $0,24,48$ or $72 \mathrm{~h}$ after treatment using the CellTiter-Glo luminescent cell viability assay. Data represent the mean $\pm \mathrm{SD}$ of three independent experiments.

\section{A ORF62}

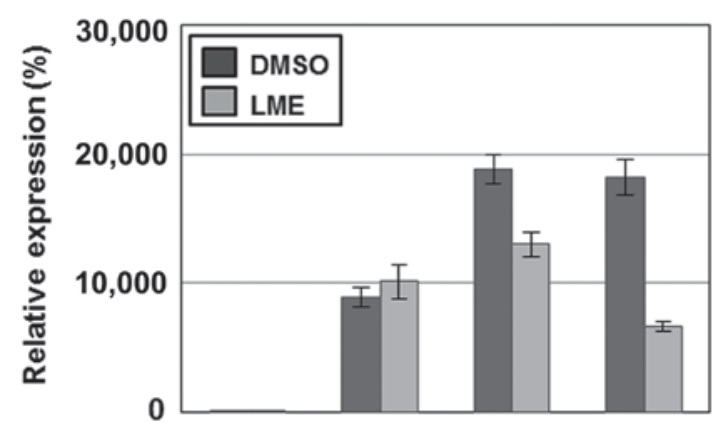

\section{B ORF28}

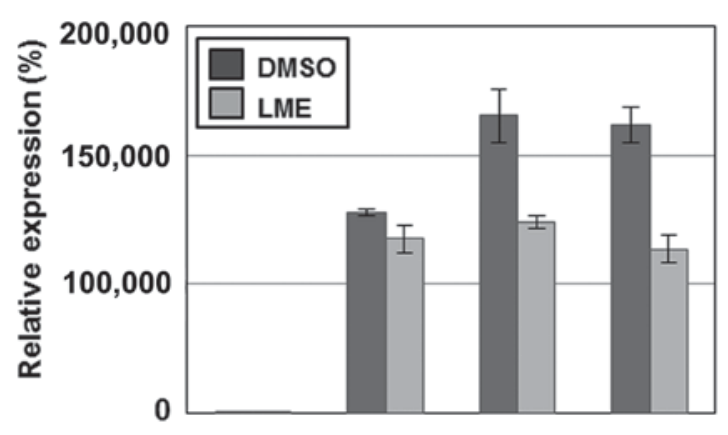

C $g B$

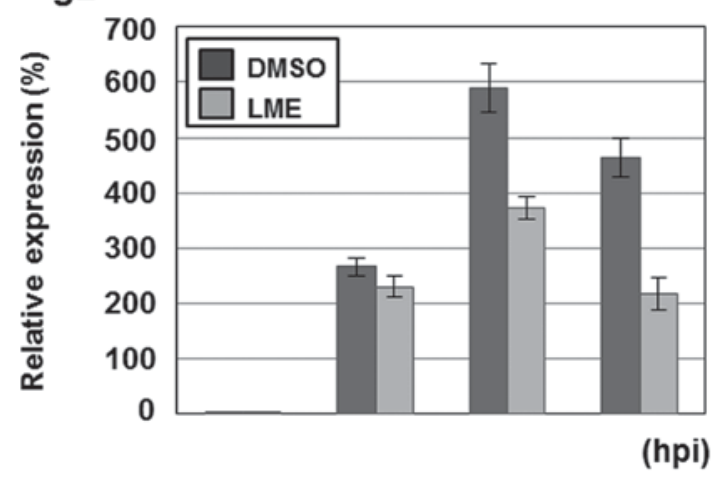

Figure 4. Effects of LME on VZV lytic gene expression. MRC-5 cells were inoculated with VZV-YC01-infected MRC-5 cells at an MOI of 0.1 and treated with either DMSO or LME at a concentration of $25 \mu \mathrm{g} / \mathrm{ml}$. At 0,24 , 48 or $72 \mathrm{~h}$ after infection, total RNA was harvested and reverse-transcribed into cDNA. The relative amounts of (A) ORF62, (B) ORF28 and (C) gB transcripts were assessed via qRT-PCR, as described in Materials and methods.

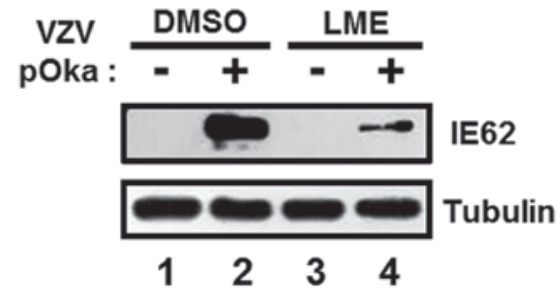

Figure 5. Downregulation of VZV IE gene expression by LME. MRC-5 cells were inoculated with VZV-pOka-infected MRC-5 cells at an MOI of 0.1 and treated with either DMSO or LME at a concentration of $25 \mu \mathrm{g} / \mathrm{ml}$. At $72 \mathrm{~h}$ after infection, equal amounts of cell extracts were subjected to western blot analysis with antibodies specific for VZV IE62 or tubulin.

or LME at a concentration of $25 \mu \mathrm{g} / \mathrm{ml}$. At $72 \mathrm{~h}$ after infection, IE62 protein expression was assessed by western blot analysis with a specific anti-IE62 antibody. IE62 protein was strongly induced in DMSO-treated cells (Fig. 5; compare lanes 2 and 1), and expression was significantly reduced by LME (Fig. 5; compare lane 4 with 2). Based on the collective results, we propose that LME downregulates IE62 protein expression and, in turn, negatively affecting VZV replication in vitro.

\section{Discussion}

Varicella-zoster virus infection causes severe disease in immunocompromised individuals, especially those with impaired cell-mediated immune responses. VZV infection can develop to disseminated disease, such as widespread skin lesion, pneumonia, hepatitis, or encephalitis (3).

Owing to side effects and emergence of resistant VZV strains, limited antiviral drugs are currently available to VZV-associated diseases $(7,9)$. Plant extracts have been utilized extensively to develop novel therapeutic agents that induce fewer side effects than synthetic drugs (4). The main objective of the present study was to investigate the antiviral activities of plant extracts against VZV.

In LME-treated MRC-5 cells, replication of both laboratory and clinical strains of VZV was significantly inhibited. Since LME did not exert significant adverse effects on MRC5 cell viability, its anti-VZV activity does not appear to be attributable to cytotoxicity. Lysimachia mauritiana is a biennial herb distributed worldwide, mainly in the temperate and subtropical climate regions of both hemispheres, along coastal regions in East Asia, the Philippines, Micronesia, Polynesia and the Indian Ocean islands (13). The chemical constituents and biological activities of Lysimachia mauritiana have not been investigated to date.

Our data indicate that LME reduces VZV lytic gene expression and inhibits VZV replication. LME induced significant downregulation of IE62 protein, an important transactivation of IE, $\mathrm{E}$ and $\mathrm{L}$ genes $(2,3)$, in turn, leading to suppression of $\mathrm{E}$ and $\mathrm{L}$ genes and inhibition of VZV replication.

The mechanisms underlying LME-mediated downregulation of VZV IE62 protein expression remain to be elucidated. Interestingly, LME suppressed the expression of lytic genes at later stages of VZV infection to a significant extent, but had almost no effect on lytic gene expression at the earlier time points (Fig. 4; compare 24 with 48 and 72 hpi). At the earlier stages of infection, pre-generated IE62 protein in tegument may 
induce VZV lytic genes. LME may reduce lytic gene expression by downregulating IE62 protein expression, consequently leading to suppression of lytic gene expression at subsequent time-points of VZV infection. LME may either directly inhibit the activities of transcription factors or indirectly interfere with a signal transduction pathway(s) to activate transcription factors for IE62 expression. In addition to downregulation of IE62 protein expression, it is possible that LME affects other phases of the VZV life cycle.

To our knowledge, this is the first study to demonstrate that LME exhibits antiviral activity against VZV. LME may provide an effective source for further development and tailoring of novel therapeutic agents to treat VZV-associated diseases.

\section{Acknowledgements}

We thank Dr Chan-Hee Lee (Chungbuk National University, Cheongju, Korea) for kindly providing the pOka and YC01 strains of VZV. We are grateful to Dr Jin-Hyun Ahn (Sungkyunkwan University, Suwon, Korea) for helpful discussions. This study was supported by the Bio-industry Technology Development Program, Ministry of Agriculture, Food and Rural Affairs (no. 311063-5) and the Gachon University Research Fund of 2014 (GCU-2014-0198).

\section{References}

1. Arvin AM and Gilden D: Varicella-zoster virus. In: Fields Virology. Knipe DM and Howley PM (eds). 6th edition. Lippincott Williams \& Wilkins, Philadelphia, PA, pp2015-2057, 2013.

2. Zerboni L, Sen N, Oliver SL and Arvin AM: Molecular mechanisms of varicella zoster virus pathogenesis. Nat Rey Microbiol 12: 197-210, 2014.
3. Cohen JI, Brunell PA, Straus SE and Krause PR: Recent advances in varicella-zoster virus infection. Ann Intern Med 130: 922-932, 1999.

4. To KP,Kang SC and Song YJ: The extract of Elaeocarpussylvestris inhibits human cytomegalovirus immediate early gene expression and replication in vitro. Mol Med Rep 9: 744-748, 2014.

5. Arvin AM: Varicella-zoster virus. Clin Microbiol Rev 9: 361-381, 1996.

6. Zerboni L, Ku CC, Jones CD, Zehnder JL and Arvin AM: Varicella-zoster virus infection of human dorsal root ganglia in vivo. Proc Natl Acad Sci USA 102: 6490-6495, 2005.

7. Sasivimolphan P, Lipipun V, Likhitwitayawuid K, Takemoto M, Pramyothin P, Hattori M and Shiraki K: Inhibitory activity of oxyresveratrol on wild-type and drug-resistant varicella-zoster virus replication in vitro. Antiviral Res 84: 95-97, 2009

8. Docherty JJ, Sweet TJ, Bailey E, Faith SA and Booth T: Resveratrol inhibition of varicella-zoster virus replication in vitro. Antiviral Res 72: 171-177, 2006.

9. Choi EJ, Lee CH, Kim YC and Shin OS: Wogonin inhibits Varicella-Zoster (shingles) virus replication via modulation of type I interferon signaling and adenosine monophosphateactivated protein kinase activity. J Funct Foods 17: 399-409, 2015.

10. Gilden D, Mahalingam R, Nagel MA, Pugazhenthi S and Cohrs RJ: Review: The neurobiology of varicella zoster virus infection. Neuropathol Appl Neurobiol 37: 441-463, 2011.

11. Won YH, Kim JI, Kim YY and Lee CH: Characterization of the repeat sequences of varicella-zoster virus. J Bacteriol Virol 44: 326-335, 2014

12. Kim SY, Kim JE, Won J and Song YJ: Characterization of the rapamycin-inducible EBV LMP1 activation system. J Microbiol 53: 732-738, 2015.

13. Kono Y, Chung KF, Chen CH, Hoshi Y, Setoguchi H, Chou $\mathrm{CH}$, Oginuma $\mathrm{K}$ and Peng $\mathrm{CI}$ : Intraspecific karyotypic polymorphism is highly concordant with allozyme variation in Lysimachia mauritiana (Primulaceae: Myrsinoideae) in Taiwan: implications for the colonization history and dispersal patterns of coastal plants. Ann Bot 110: 1119-1135, 2012. 